\title{
ZFPM2-AS1 promotes the proliferation, migration, and invasion of human non-small cell lung cancer cells involving the JAK-STAT and AKT pathways
}

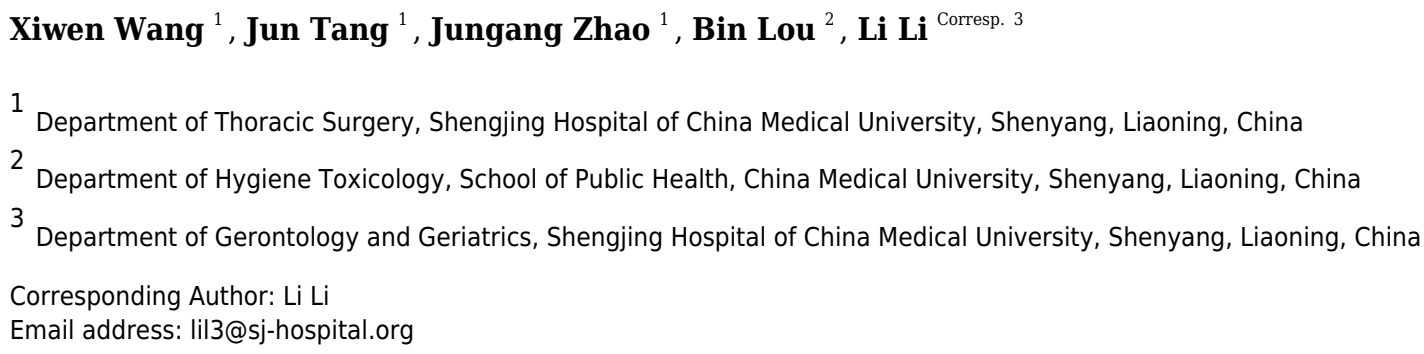

Purpose: Recent studies have determined that long non-coding RNAs (IncRNAs) are potential prognostic biomarkers for non-small cell lung cancers (NSCLCS). The purpose of this study was to analyze the function and associated pathways of zinc finger protein multitype 2 antisense RNA 1 (ZFPM2-AS1) in NSCLC cells. Methods: We used qRT-PCR to analyze ZFPM2-AS1's transcription level. Its proliferation, migration, and invasion capacities were determined using MTT, colony forming, wound healing, and transwell assays. We additionally analyzed the correlation between ZFPM2 and immune infiltration using the Tumor Immune Estimation Resource (TIMER) database, and the protein expression levels using Western blots. Results: We found that ZFPM2-AS1 expression in NSCLC specimens and cell lines was elevated compared to the control group. ZFPM2-AS1 is an oncogene and independent prognostic predictor of poor survival in NSCLCs, and its expression had a positive correlation with tumor size and lymph node metastasis in our clinical data. MTT, colony forming, wound healing, and transwell assays showed a positive correlation between ZFPM2-AS1 expression and the proliferation, migration, and invasion of NSCLC cells in the presence and absence of interferon-y (IFN- $\gamma$ ). Using the TIMER database, we hypothesized that ZFPM2 was negatively correlated with ZFPM2-AS1 expression, as well as the immune infiltration levels in lung adenocarcinoma (LUAD). Finally, we found that ZFPM2-AS1 negatively regulated ZFPM2 expression, and had a positive correlation with PD-L1 expression through the JAK-STAT and AKT pathways.

Conclusion: Our study confirmed that ZFPM2-AS1 promotes the proliferation, migration, and invasion of NSCLC cells via the JAK-STAT and AKT pathways. Further research on the ZFPM2-AS1 pathway regulation mechanism is needed. 
1 ZFPM2-AS1 promotes the proliferation, migration, and invasion of human non-

2 small cell lung cancer cells involving the JAK-STAT and AKT pathways

3 XI-WEN WANG ${ }^{1}$, JUN TANG ${ }^{1}$, JUN-GANG ZHAO ${ }^{1}$, BIN LOU ${ }^{3}$, LI LI $^{2}$,*

5 Xiwen Wang (wangxw@sj-hospital.org)

$6 \quad$ Jun Tang (jtang@cmu.edu.cn)

7 Jun-Gang Zhao (zhaojg@sj-hospital.org)

8 Bin Lou (binlougg@gmail.com)

9 Li Li (lil3@sj-hospital.org)

$11{ }^{1}$ Department of Thoracic Surgery, Shengjing Hospital of China Medical University, Shenyang, 12 China

13 2Department of Gerontology and Geriatrics, Shengjing Hospital of China Medical University, 14 Shenyang, China

15 32Department of Hygiene Toxicology, School of Public Health, China Medical University, 16 Shenyang, China

18 *Correspondence should be addressed to:

19 LiLi(lil3@sj-hospital.org)

20 Department of Gerontology and Geriatrics, Shengjing Hospital of China Medical University, 21 Shenyang, China

22 Email: li13@sj-hospital.org

\section{Abstract}

25 Purpose: Recent studies have determined that long non-coding RNAs (lncRNAs) are potential 26 prognostic biomarkers for non-small cell lung cancers (NSCLCs). The purpose of this study was 
27 to analyze the function and associated pathways of zinc finger protein multitype 2 antisense RNA 281 (ZFPM2-AS1) in NSCLC cells.

29 Methods: We used qRT-PCR to analyze ZFPM2-AS1's transcription level. Its proliferation, 30 migration, and invasion capacities were determined using MTT, colony forming, wound healing, 31 and transwell assays. We additionally analyzed the correlation between ZFPM2 and immune 32 infiltration using the Tumor Immune Estimation Resource (TIMER) database, and the protein 33 expression levels using Western blots.

34 Results: We found that ZFPM2-AS1 expression in NSCLC specimens and cell lines was elevated 35 compared to the control group. ZFPM2-AS1 is an oncogene and independent prognostic predictor 36 of poor survival in NSCLCs, and its expression had a positive correlation with tumor size and 37 lymph node metastasis in our clinical data. MTT, colony forming, wound healing, and transwell assays showed a positive correlation between ZFPM2-AS1 expression and the proliferation, migration, and invasion of NSCLC cells in the presence and absence of interferon- $\gamma$ (IFN- $\gamma$ ). Using the TIMER database, we hypothesized that ZFPM2 was negatively correlated with ZFPM2AS1 expression, as well as the immune infiltration levels in lung adenocarcinoma (LUAD). Finally, we found that ZFPM2-AS1 negatively regulated ZFPM2 expression, and had a positive correlation with PD-L1 expression through the JAK-STAT and AKT pathways.

\section{Introduction}

Lung cancer is the most deadly malignant tumor, accounting for more than $80 \%$ of non-small cell lung cancers (NSCLCs) worldwide, most of which are lung adenocarcinoma (LUAD) and lung squamous cell carcinoma (LUSC). Multiple therapies have improved the prognosis of NSCLCs, 
53 but the 5-year survival rate has remained lower than $20 \%$ (1). Therefore, it is crucial to explore 54 novel diagnostic biomarkers and therapeutic approaches $(2,3)$.

55 Long non-coding RNAs (lncRNAs), defined as non-coding transcripts longer than 200 56 nucleotides, are associated with the initiation, progression, and prognosis of various tumors $(4,5)$.

57 Mounting dysregulated lncRNAs may also play a role as tumor suppressors or oncogenes in 58 multiple tumors, including NSCLCs.

59 We previously screened differential expression lncRNAs in NSCLCs using The Cancer 60 Genome Atlas (TCGA) database (6). We speculated that one of the most distinct lncRNAs, zinc 61 finger protein multitype 2 antisense RNA 1 (ZFPM2-AS1), may play an important role in the 62 biological behavior of NSCLC cells. In this study, we concentrated on the functions of ZFPM263 AS1. ZFPM2-AS1 reportedly induces p53 destabilization by stabilizing macrophage migration 64 inhibitory factor (MIF), leading to the progression of gastric cancer (7). ZFPM2-AS1 has also been shown to promote metastasis and proliferation, as well as inhibit renal cell cancer apoptosis by targeting miR-137 (8). Additionally, ZFPM2-AS1 can promote NSCLC progression via the miR511-3p/AFF4 and miR-18b-5p/VMA21 pathways $(9,10)$, and enhance the malignancy of cervical cancer by sponging microRNA-511-3p (11). By upregulating TRAF4, ZFPM2-AS1 facilitates cell proliferation in both esophageal squamous cell carcinoma and small cell lung cancer $(12,13)$. However, ZFPM2-AS1's molecular regulatory network in NSCLC cells remains unclear.

The PI3K/AKT and JAK/STAT signaling pathways promote cell proliferation and motility by activating key metastasis-promoting genes (14). STAT activation is restricted in normal cells. 73 However, once STAT is activated, numerous genes that control tumor cell proliferation, angiogenesis, and evasion of immune surveillance are uncontrollably expressed (15). Interferon- $\gamma$ (IFN- $\gamma$ ) is crucial for immunity against intracellular pathogens and tumor cells (16). Since IFN- $\gamma$ has the ability to induce PD-L1, IFN- $\gamma$ expression in cancer cells may weaken the immunity of specific tumor cells (17). Additionally, it has been found that PD-L1 expression is positively 
correlated with JAK2 in NSCLCs via the JAK-STAT axis (18). However, it has not been proved whether ZFPM2-AS1 can regulate PD-L1 via the JAK-STAT and AKT pathways.

In this study, we investigated ZFPM2-AS1's proliferation, migration, and invasion abilities in NSCLC cells. We also determined the regulatory roles of ZFPM2-AS1 in the JAK-STAT and AKT pathways.

\section{Materials and methods}

\section{1 | TCGA and Tumor Immune Estimation Resource (TIMER) databases}

We used ZFPM2-AS1 transcript expression levels extracted from TCGA's database for our fragments per kilobase million (FPKM) values. The FPKM values were plotted in a scatterplot and on receiver operating characteristic (ROC) curves. We used OmicShare tools (http://www. omicshare. com/tools) to find the area under the ROC curve (AUC) in order to estimate the diagnostic values (sensitivity and specificity). TIMER (https://cistrome.shinyapps.io/timer) is a novel database that includes 10,897 samples across 39 tumor types from TCGA (19), along with specific genes' tumor immune infiltration levels. We analyzed ZFPM2 expression across multiple tumor types using the different expression module, and identified the association between ZFPM2 expression and immune infiltration level using the gene module.

\section{2 | Patients and samples}

Surgical specimens were collected from 50 individual patients undergoing NSCLC surgery at the Affiliated Shengjing Hospital of China Medical University (Shenyang, China) between May 2017 and August 2018. All specimens had been pathologically diagnosed as LUAD or LUSC. The specimens were frozen at $-80^{\circ} \mathrm{C}$ directly following surgery. Our experimental protocol was authorized by the Shengjing Hospital Ethics Committee (2018PS170K), and we acquired written informed consent from each patient.

\section{3 | Cell culture, reagent, and transfection}


The human NSCLC cell lines (A549 and H460) were purchased from the Shanghai Institutes of Biochemistry and Cell Biology, Chinese Academy of Sciences, Shanghai, China. The A549 and H460 cell lines had been cultured in RPMI-1640 with 10\% fetal bovine serum (Clark Biosciences, Richmond, VA, USA), $100 \mathrm{U} / \mathrm{ml}$ penicillin, and $100 \mathrm{ug} / \mathrm{ml}$ streptomycin (Sigma-Aldrich, St. Louis, MO, USA) in a $5 \% \mathrm{CO}_{2}$ incubator at $37^{\circ} \mathrm{C}$. During IFN- $\gamma$ stimulation, cells were incubated with $100 \mathrm{ng} / \mathrm{ml}$ of recombinant human IFN- $\gamma$ (Peprotech, Cranbury, NJ, USA) for 48 hrs. We used

110 Lipo3000 (Invitrogen, Carlsbad, CA, USA) according to our transfection protocol. We used 20

$111 \mathrm{uM}$ of lncRNA Smart Silencer (RiboBio, Guangzhou, China) and a mixture of three siRNAs and

112 three antisense oligonucleotides. The sequences are provided in Table S1. ZFPM2-AS1

113 overexpression plasmid was provided by GenePharma (Shanghai, China).

\section{$114 \quad 2.4 \mid$ RNA isolation, cDNA synthesis, and quantitative real-time RT-PCR}

115 We extracted total RNA using TRIzol reagent, and performed reverse-transcription using 116 HisScript ${ }^{\mathrm{TM}}$ QRT SuperMix (Vazyme Biotech Co., Ltd., Nanjing, China). We used qRT-PCR and 117 ChamQ ${ }^{\mathrm{TM}}$ Universal SYBR qPCR Master Mix (Vazyme Biotech Co., Ltd.) to analyze the relative 118 expression of the control group. The specific primers are shown in Table S1. GAPDH and RPS18

119 were used as housekeeping genes. The relative gene expression was calculated using the $2^{-\Delta \Delta \mathrm{Cq}}$ 120 method.

\section{$121 \quad 2.5 \mid$ MTT assay}

122 Cell viability was determined using MTT reagent (Sigma-Aldrich) with a concentration of $1230.5 \mathrm{mg} / \mathrm{mL}$ for $4 \mathrm{~h}$. We seeded $4 \times 10^{3}$ cells per well in 96-well plates for $0,1,2,3,4$, and 5 days.

124 The supernatant was abandoned, and we precipitated formazan with DMSO. Finally, we analyzed 125 the absorbance at $450 \mathrm{~nm}$ using a microplate reader (Thermo Fisher Scientific, Waltham, MA, 126 USA).

\section{$127 \quad 2.6 \mid$ Colony formation assay}

128 We cultured about 3,000 terminated trypsinized cells in 6-cm dishes three times at $37^{\circ} \mathrm{C}$ in $1295 \% \mathrm{CO}_{2}$. Two weeks later, the cell colonies were fixed with $10 \%$ methanol for $30 \mathrm{~s}$, and then 
130 stained with $0.1 \%$ crystal violet (Sigma-Aldrich) for $15 \mathrm{~min}$. Finally, the visible colonies were

131 counted using a microscope.

$132 \quad \mathbf{2 . 7} \mid$ Wound healing assay

133 A549 and H460 cells were seeded into $5 \times 10^{5}$ cells/well in six-well plates three times. A 200

$134 \mu \mathrm{L}$ pipette tip was used to make a scratch where the confluence reached $90 \%$. The cells were then

135 incubated at $37^{\circ} \mathrm{C}$ in $5 \% \mathrm{CO}_{2}$ for $24 \mathrm{~h}$. We studied the migration distances using an FSX100

136 Biological Image system (Olympus, Tokyo, Japan).

\section{$137 \quad \mathbf{2 . 8} \mid$ Transwell migration and invasion assay}

138 Migration and invasion assays were placed in 24-well Transwell chambers that had $8 \mu \mathrm{m}$ size

139 pores (Costar, Washington, D.C., USA). In the invasion assay, the pores were covered with 100

$140 \mu \mathrm{L}$ of Matrigel (BD Biosciences, San Jose, CA, USA). After trypsinization, we placed $100 \mu \mathrm{L}$ of

141 medium $\left(5 \times 10^{4}\right.$ cells $)$ supplemented with $2 \%$ fetal bovine serum in the upper Transwell chamber,

142 and $600 \mu \mathrm{L}$ of medium supplemented with $10 \%$ fetal bovine serum in the lower chamber. After 24

$143 \mathrm{~h}$ of incubation, the upper cells were removed, and the lower ones were fixed with

144 paraformaldehyde and stained with hematoxylin. The number of migrated/invaded cells was

145 analyzed using 10 randomly selected fields at $\times 200$ magnification under phase contrast microscopy

146 (Olympus). All assays were performed independently at least three times.

\section{$147 \quad 2.9 \mid$ Nuclear-cytoplasmic localization}

148 We harvested and washed the A549 cells before adding $500 \mu 1$ of cell disruption buffer

149 (PARIS kit; cat. no. AM1921; Invitrogen/Thermo Fisher Scientific) to the cells on ice for $10 \mathrm{~min}$.

150 Following centrifugation at $500 \mathrm{x}$ g, the supernatants were preserved as cytoplasmic RNA. They

151 were washed, an equal volume of nuclear lysate buffer was added, and they were centrifuged at

$152500 \mathrm{x}$ g. Finally, the supernatants were collected as nuclear RNA and we performed qRT-PCR

153 using the primers listed in Table S1.

$154 \quad \mathbf{2 . 1 0}$ | Flow cytometry 
155 For cell cycle analysis, we harvested the transfected cells, washed them with PBS, 156 resuspended them in $0.9 \mathrm{ml}$ of PBS, and gradually added ice-cold ethanol up to a volume of $3 \mathrm{ml}$. 157 After 24 hours of fixation, the cells were incubated with 0.1\% Triton X-100, 0.2 mg/ml RNase A, 158 and $25 \mu \mathrm{g} / \mathrm{ml}$ propidium iodide (PI) for 30 minutes at room temperature. We used flow cytometry 159 to assay the DNA content (Becton Dickinson, Bedford, MA, USA), and ModFit software (Verify 160 Software) to quantify the percentage of cells within the S-, G0/G1-, and G2/M- phases of the cell 161 cycle.

\section{$162 \quad 2.11 \mid$ Western blot analysis}

163 After harvesting the proteins using a lysis buffer $(50 \mathrm{mM}$ of Tris $(\mathrm{pH} 7.4), 1 \%$ Triton $\mathrm{X}-100$, $1640.5 \%$ Nonidet P-40, $150 \mathrm{mM} \mathrm{NaCl}$, and protease inhibitor), we separated them using $10 \%$ sodium

165 dodecyl sulfate (SDS) polyacrylamide gel electrophoresis. The proteins were then transferred to 166 polyvinylidene difluoride (PVDF) membranes (Millipore, Burlington, MA, USA) for 2 h at 220 $167 \mathrm{~mA}$. The membranes were blocked in 5\% BSA with TBST for $1 \mathrm{~h}$ at room temperature, and were 168 incubated overnight using primary antibodies at $4^{\circ} \mathrm{C}$. We incubated the secondary antibody with 169 horseradish peroxidase (HRP) conjugates for $1 \mathrm{~h}$ at room temperature. We identified the bands 170 using a chemiluminescence detection kit (Tanon, Shanghai, China). We found that the primary 171 antibodies included PDL1 (E1L3N), JAK2 (D2E12), phospho-STAT3 (Tyr705; D3A7), STAT3 172 (79D7), phospho-AKT (Ser473; D9E), AKT (40D4; all purchased from Cell Signaling 173 Technology, Boston, MA, USA), and ZFPM2 (OriGene).

\section{$174 \quad \mathbf{2 . 1 2} \mid$ Statistical analysis}

175 The log-rank test was performed using the Kaplan-Meier survival analysis procedure (16).

176 We conducted statistical analyses with GraphPad Prism7 (GraphPad Software, Inc., San Diego, 177 CA, USA). Test data were manifested as means \pm standard deviation (SD). We used the student's 178 t-test (two-tailed) to find the differences between two groups, and one-way ANOVA to find the 179 differences across more than two groups, followed by Dunnett's post-test. $\mathrm{P}<0.05$ was considered 180 statistically significant. 


\section{Results}

3.1 Increased ZFPM2-AS1 related to poor survival in in NSCLC patients

During our investigation, we used ZFPM2-AS1 RNA-seq data from TCGA to analyze the

185

186

187

188

189

190

191

192

193

194

195

196

197

198

199

200

201

202

203

204

205

206 differential expressions across 59 normal lung tissues and 535 LUADs or LUSCs. We found a significant increase of ZFPM2-AS1 in NSCLCs compared to normal lung tissues (Fig. 1A). Furthermore, when analyzing the ROC curve based on the screened TCGA data, we found that ZFPM2-AS1's AUC value was 0.891 (Fig. 1B), indicating that ZFPM2-AS1 may be a novel diagnostic biomarker. Generated using screened data from TCGA, the Kaplan-Meier survival curve showed that higher ZFPM2-AS1 expression levels were significantly associated with poor prognoses for NSCLC patients (Fig. 1C). Ultimately, our results suggested that ZFPM2-AS1 is a possible oncogene in NSCLCs.

3.2 Validating ZFPM2-AS1 expression patterns in NSCLC samples and cell lines

To further verify the ZFPM2-AS1 expression patterns that we found in TCGA's RNA-Seq databases, we used qRT-PCR to validate 50 pairs of collected NSCLC and adjacent normal samples. We found that the relative transcription expression levels were significantly higher in NSCLC tissue compared to the adjacent normal tissues (Fig. 2A; $<<0.0001$ ). We also validated BEAS-2B, A549, NCI-H460, H1299, H292, and HEK293 cells by performing qRT-PCR, and found that ZFPM2-AS1 was expressed in all of these cell lines. Compared to the BEAS-2B cell expression levels, ZFPM2-AS1 was significantly upregulated in the A549 and H460 cells. The expression levels were significantly downregulated in the NSCLC cell lines H1299 and H292 (Fig. 2B). These results implied that ZFPM2-AS1's function in A549 and H460 cells may be similar to its functions in NSCLC samples.

3.3 The association between ZFPM2-AS1 and NSCLC clinical characteristics When examining the possible association between ZFPM2-AS1 expression levels and the clinical parameters of 50 NSCLC patients, we found that tumor size was significantly positively 
207

208

209

210

211

212

213

214

215

216

217

218

219

220

221

222

223

224

225

226

227

228

229

230

231

232

correlated with ZFPM2-AS1 expression $(\mathrm{p}=0.007)$. The tumor-node-metastasis (TNM) stage was also positively correlated with ZFPM2-AS1 expression $(\mathrm{p}=0.047)$. However, ZFPM2-AS1 expression did not have a significant correlation with other clinical characteristics, including age, gender, tumor differentiation, smoking history, and lymph node metastasis (Table 1). Our results showed that ZFPM2-AS1 expression was positively correlated to tumor size and TNM stage.

3.4 ZFPM2-AS1 knockdown decreases proliferation and colony formation in NSCLC cell lines

Since ZFPM2-AS1 was highly expressed in A549 cells, we used siRNA-mediated ZFPM2AS1 knockdown in A549 cells to analyze ZFPM2-AS1's biological functions. To minimize the off-target effects on lncRNA, we used specific Smart Silencers, including three individual siRNAs and three individual antisense oligonucleotides. The decreased efficiency was confirmed by qRTPCR (Fig. 3A). Subsequently, we used an MTT assay to show any cell proliferation caused by ZFPM2-AS1 silencing or overexpression. We also stimulated ZFPM2-AS1 using IFN- $\gamma$ because of its ability to induce PD-L1 and promote the immune escape of tumor cells. The results showed that the proliferation rate of ZFPM2-AS1 silencing in the H460 cells was aberrantly lower than in the control group after 72-120 hours (Fig. 3B). In the A549 cells, the proliferation rate was significantly lower when silencing ZFPM2-AS1 after 72 hours without IFN- $\gamma$, and after 96-120 hours with IFN- $\gamma$ (Fig. 3C). We obtained similar results when performing colony forming assays, confirming that ZFPM2-AS1 knockdown distinctly inhibited colony forming in both A549 and H460 cells (Fig. 3D-G). Meanwhile, ZFPM2-AS1 overexpression significantly promoted A549 cell proliferation after 96-120 hours without IFN- $\gamma$, and after 72-120 hours with IFN- $\gamma$ (Fig. 3H).

These results suggest that ZFPM2-AS1's role as an oncogene involves promoting NSCLC cell line proliferation.

3.5 $\mid$ ZFPM2-AS1 promotes the migration and invasion of NSCLC cell lines

The results of the wound healing assay indicated that ZFPM2-AS1 knockdown significantly inhibited A549 and H460 cell motility when compared to the control group (Fig. 4A-D). We used the Transwell assay to investigate whether migration and invasion were also affected by ZFPM2- 
233

234

235

236

237

238

239

240

241

242

243

244

245

246

247

248

249

250

251

252

253

254

255

256

257

258

AS1 in NSCLC cells. We found that the siRNA-mediated ZFPM2-AS1 knockdown significantly inhibited the invasion capacities of both A549 and H460 cells (Fig. 4E, F). The silencing of ZFPM2-AS1 distinctly impeded the invasion and migration capabilities of A549 cells, in both the presence and absence of IFN- $\gamma$ (Fig. 4G-J). Meanwhile, ZFPM2-AS1 overexpression significantly promoted invasion and migration in the presence or absence of IFN- $\gamma$ (Fig. 4K-N). Ultimately, we determined that ZFPM2-AS1 promoted migration and invasion in both A549 and H460 cells.

3.6 ZFPM2-AS1's primary expression in the nucleus did not affect the A549 cell cycle

To clarify its cellular localization, we used qRT-PCR to determine ZFPM2-AS1 expression in both the nuclear and cytoplasmic fractions of A549 cells. We used GAPDH expression for the cytoplasmic indicator, and U1 snRNA enrichment for the nuclear indicator. The results showed that ZFPM2-AS1 was primarily expressed in the nucleus (Fig. 5A), indicating that ZFPM2-AS1 may also regulate the functions of NSCLC cells in the nucleus. We used a flow cytometric analysis to determine whether ZFPM2-AS1 silencing or overexpression had an impact on the cell cycle, with and without IFN- $\gamma$. The results showed that IFN- $\gamma$ arrested A549 cells at the G0/G1 phase. However, ZFPM2-AS1 silencing and overexpression did not significantly affect the A549 cell cycle (Fig. 5B-I). Our results confirmed that ZFPM2-AS1 was primarily expressed in the nucleus, but its effect on the proliferation, migration, and invasion of A549 cells was not dependent on the cell cycle.

3.7 The association between ZFPM2, a potential target for ZFPM2-AS1, and LUAD tumor immune infiltration level

To determine whether ZFPM2-AS1 was associated with tumor immune infiltration level, we mined data from the TIMER database. Since ZFPM2 is a potential target for ZFPM2-AS1, we found that ZFPM2 expression was significantly lower in most human tumors compared to the adjacent normal tissues, including LUAD and LUSC (Figure 6A). ZFPM2-AS1 expression was significantly higher in NSCLC tissues compared to normal lung tissues (Fig. 1A), suggesting a possible negative correlation between ZFPM2-AS1 and ZFPM2. We also found a negative 
259

260

261

262

263

264

265

266

267

268

269

270

271

272

273

274

275

276

277

278

279

280

281

282

283

284

correlation between ZFPM2 expression and the immune infiltrate levels of tumor purity $(\mathrm{R}=-$ 0.352, $\mathrm{P}=8.14 \mathrm{e}^{-16}$ ) in LUAD (Figure 6B). These results implied that ZFPM2 expression was significantly lower in LUAD and LUSC, as well as negatively correlated with tumor immune infiltration levels. We wanted to further verify the positive correlation between ZFPM2-AS1 and the immune infiltrating marker PD-L1 using Western blot.

3.8 ZFPM2-AS1's negative regulation of ZFPM2 expression and positive regulation of PD-L1 expression via the JAK-STAT and AKT pathways

First, we silenced ZFPM2-AS1 expression using three individual siRNAs in the A549 cells.

The results showed a significant increase in ZFPM2 expression, indicating that ZFPM2-AS1 may negatively regulate ZFPM2 expression. Meanwhile, JAK2, p-STAT3, and p-AKT expression decreased in comparison to the control group. However, there was no significant differential STAT3 and AKT expression (Fig. 7A, B). We used siRNA to induce more significant ZFPM2 differences in the following experiment. We performed ZFPM2-AS1 silencing, both with and without IFN- $\gamma$ exposure, to identify the differences between the JAK-STAT and AKT pathways. We found that in both the presence and absence of IFN- $\gamma$, ZFPM2-AS1 knockdown significantly upregulated ZFPM2 expression and downregulated JAK2, p-STAT3, and p-AKT expression. PDL1 expression was distinctly inhibited when ZFPM2-AS1 was silenced by IFN- $\gamma$ stimulation (Fig. 7C, D). Furthermore, ZFPM2-AS1 overexpression downregulated ZFPM2 expression and upregulated JAK2, p-STAT3, p-AKT, and PD-L1 expression in the presence or absence of IFN- $\gamma$ (Fig. 7E, F). Ultimately, we determined that ZFPM2-AS1 negatively regulated ZFPM2 expression and positively regulated PD-L1 expression through the JAK-STAT and AKT pathways.

\section{Discussion}

Recent studies have shown that IncRNAs can operate as biomarkers in the diagnosis, therapy, and prognosis of various malignant tumors $(20,21)$. Our results indicated that ZFPM2-AS1 was upregulated in NSCLC specimens and cell lines when compared to the control groups. 
285

286

287

288

289

290

291

292

293

294

295

296

297

298

299

300

301

302

303

304

305

306

307

308

309

310

Additionally, we found a correlation between ZFPM2-AS1 and poor survival in TCGA. When looking at clinical statistics, we found that higher ZFPM2-AS1 expression levels were positively correlated with larger tumor sizes and later TNM stages. These findings suggest that ZFPM2-AS1 may be a potential novel biomarker for NSCLC. Our study also verified that the downregulation of ZFPM2-AS1 expression significantly inhibited the proliferation, migration, and invasion of A549 and H460 cells, suggesting that ZFPM2-AS1 frequently played an oncogenic role. Similarly, Han et al. (2020) reported that ZFPM2-AS1 facilitated the proliferation, invasion, and epithelialto-mesenchymal transition in LUAD, and that UPF1 de-stabilized the ZFPM2 mRNA level negatively regulated by ZFPM2-AS1. Their observation of a negative correlation between ZFPM2-AS1 and ZFPM2 was also consistent with our findings.

ZFPM2-AS1 has been reported to induce p53 destabilization stabilizing MIF, leading to the progression of gastric cancer (7). However, the connection between ZFPM2-AS1 and the JAK/STAT signal pathway has not been explored. In our study, we found that ZFPM2-AS1 positively regulated the expression of JAK2, p-STAT3, and PD-L1 in A549 cells. JAK kinase phosphorylated STAT C-terminus Tyr705 in STAT3, initiated by the binding of IL-6 to its specific receptor and the activation of phosphorylated JAK. A different study reported that the activation of p-STAT3 enhanced cell proliferation, metastasis, and angiogenesis in multiple cancers including NSCLC (23). PI3K/AKT, RAS/MAPK, and JAK/STAT3 are three major downstream activated EGFR phosphorylation pathways (24). Our results revealed that ZFPM2-AS1 also positively regulated p-AKT expression, confirming the existence of crosstalk between the JAK2STAT3 and PI3K-AKT pathways.

Previous research has shown that PD-L1 expression is involved in two main mechanisms: the innate immune escape, which is associated with multiple oncogenes, and the adaptive immune escape, which consists of various tumor microenvironment inflammatory factors (25-28). Wang et al. (2019) reported that the lncRNA MALAT1 regulated PD-L1 by sponging miR-195 in diffuse large B cell lymphoma, affecting PD-L1's proliferation, apoptosis, migration, and immune escape 
311 capacities. Notably, our study confirmed that ZFPM2-AS1 knockdown decreased PD-L1

312 expression in the presence of IFN- $\gamma$, suggesting that ZFPM2-AS1 may be a potential target during

313 PD-L1 immunotherapy. However, our study's exploration of how ZFPM2-AS1 regulates PD-L1

314 in NSCLC cells was limited, and this mechanism should be thoroughly studied in future

315 investigations.

316 ZFPM2's role as a cytokine has been shown to play a crucial role in the regulation of the

317 immune system (30). Our study demonstrated that ZFPM2 expression was negatively regulated by

318 ZFPM2-AS1, indicating that ZFPM2 may also be correlated with tumor immune infiltration. The

319 TIMER database showed that ZFPM2 expression had a negative correlation with the immune 320 infiltrating levels of tumor purity in LUAD, which was consistent with our initial hypothesis.

321 However, we found that ZFPM2-AS1 was downregulated in H1299 and H292 cell lines, 322 revealing that low ZFPM2-AS1 expression may progress cancer using other signal pathways. 323 Further investigations are needed to determine how ZFPM2-AS1 regulates NSCLC function in 324 these cell lines.

3255 | Conclusion

326 In this study, we found that the lncRNA ZFPM2-AS1 functioned as an oncogene by 327 promoting the proliferation, migration, and invasion of NSCLC cells. Furthermore, we determined 328 that ZFPM2-AS1 positively regulated PD-L1 expression via the JAK-STAT and AKT pathways 329 in A549 cell lines.

330

\section{Acknowledgements}

332 We thank the Medical Research Center in the Shengjing Hospital of China Medical University for 333 providing equipment, assistance, and support, as well as all the individuals who participated in this 334 study.

335

\section{References}


337 1. Hirsch FR, Scagliotti GV, Mulshine JL, Kwon R, Curran WJ, Jr., Wu YL, Paz-Ares L. Lung 338 cancer: current therapies and new targeted treatments. Lancet (London, England) 2017, 339 389(10066): 299-311.

340 2. Ma XL, Xiao ZL, Liu L, Liu XX, Nie W, Li P, Chen NY, Wei YQ. Meta-analysis of 341 circulating tumor cells as a prognostic marker in lung cancer. Asian Pacific journal of cancer 342 prevention: APJCP 2012, 13(4): 1137-1144.

343 3. Han B, Park D, Li R, Xie M, Owonikoko TK, Zhang G, Sica GL, Ding C, Zhou J, Magis AT, 344 Chen ZG, Shin DM, Ramalingam SS, Khuri FR, Curran WJ, Deng X. Small-Molecule Bcl2 BH4 345 Antagonist for Lung Cancer Therapy. Cancer cell 2015, 27(6): 852-863.

346 4. Du Z, Fei T, Verhaak RG, Su Z, Zhang Y, Brown M, Chen Y, Liu XS. Integrative genomic 347 analyses reveal clinically relevant long noncoding RNAs in human cancer. Nature structural \& 348 molecular biology 2013, 20(7): 908-913.

349 5. Prensner JR, Chinnaiyan AM. The emergence of IncRNAs in cancer biology. Cancer 350 discovery 2011, 1(5): 391-407.

351 6. Wang X, Su R, Guo Q, Liu J, Ruan B, Wang G. Competing endogenous RNA (ceRNA) 352 hypothetic model based on comprehensive analysis of long non-coding RNA expression in lung 353 adenocarcinoma. PeerJ 2019, 7: e8024.

3547 Kong F, Deng X, Kong X, Du Y, Li L, Zhu H, Wang Y, Xie D, Guha S, Li Z, Guan M, Xie 355 K. ZFPM2-AS1, a novel lncRNA, attenuates the p53 pathway and promotes gastric carcinogenesis 356 by stabilizing MIF. Oncogene 2018, 37(45): 5982-5996.

357 8. Liu JG, Wang HB, Wan G, Yang MZ, Jiang XJ, Yang JY. Long noncoding RNA ZFPM2358 AS1 promotes the tumorigenesis of renal cell cancer via targeting miR-137. European review for 359 medical and pharmacological sciences 2019, 23(13): 5675-5681.

360 9. Li J, Ge J, Yang Y, Liu B, Zheng M, Shi R. Long noncoding RNA ZFPM2-AS1 is involved 361 in lung adenocarcinoma via miR-511-3p/AFF4 pathway. Journal of cellular biochemistry 2020, 362 121(3): 2534-2542. 
363 10. Xue M, Tao W, Yu S, Yan Z, Peng Q, Jiang F, Gao X. lncRNA ZFPM2-AS1 promotes 364 proliferation via miR-18b-5p/VMA21 axis in lung adenocarcinoma. Journal of cellular 365 biochemistry 2020, 121(1): 313-321.

366 11. Dai J, Wei R, Zhang P, Liu P. Long Noncoding RNA ZFPM2-AS1 Enhances the Malignancy 367 of Cervical Cancer by Functioning as a Molecular Sponge of microRNA-511-3p and Consequently 368 Increasing FGFR2 Expression. Cancer Manag Res 2020, 23(12): 567-580.

369 12. Sun G, Wu C. ZFPM2-AS1 facilitates cell growth in esophageal squamous cell carcinoma via 370 up-regulating TRAF4. Bioscience reports 2020, 40(4).

371 13. Yan Z, Yang Q, Xue M, Wang S, Hong W, Gao X. YY1-induced lncRNA ZFPM2-AS1 372 facilitates cell proliferation and invasion in small cell lung cancer via upregulating of TRAF4. 373 Cancer cell international 2020, 20: 108.

374 14. Teng Y, Ross JL, Cowell JK. The involvement of JAK-STAT3 in cell motility, invasion, and 375 metastasis. Jak-stat 2014, 3(1): e28086.

376 15. Bowman T, Garcia R, Turkson J, Jove R. STATs in oncogenesis. Oncogene 2000, 19(21): $377 \quad 2474-2488$.

378 16. Schoenborn JR, Wilson CB. Regulation of interferon-gamma during innate and adaptive 379 immune responses. Advances in immunology 2007, 96: 41-101.

380 17. Abiko K, Matsumura N, Hamanishi J, Horikawa N, Murakami R, Yamaguchi K, Yoshioka Y, 381 Baba T, Konishi I, Mandai M. IFN- $\gamma$ from lymphocytes induces PD-L1 expression and promotes 382 progression of ovarian cancer. British journal of cancer 2015, 112(9): 1501-1509.

383 18. Ikeda S, Okamoto T, Okano S, Umemoto Y, Tagawa T, Morodomi Y, Kohno M, Shimamatsu 384 S, Kitahara H, Suzuki Y, Fujishita T, Maehara Y. PD-L1 Is Upregulated by Simultaneous 385 Amplification of the PD-L1 and JAK2 Genes in Non-Small Cell Lung Cancer. Journal of thoracic 386 oncology : official publication of the International Association for the Study of Lung Cancer 2016, 387 11(1): 62-71. 
388 19. Li T, Fan J, Wang B, Traugh N, Chen Q, Liu JS, Li B, Liu XS. TIMER: A Web Server for 389 Comprehensive Analysis of Tumor-Infiltrating Immune Cells. Cancer research 2017, 77(21): 390 e108-e110.

391 20. Heery R, Finn SP, Cuffe S, Gray SG. Long Non-Coding RNAs: Key Regulators of Epithelial392 Mesenchymal Transition, Tumour Drug Resistance and Cancer Stem Cells. Cancers 2017, 9(4).

393 21. Chandra Gupta S, Nandan Tripathi Y. Potential of long non-coding RNAs in cancer patients: 394 From biomarkers to therapeutic targets. International journal of cancer 2017, 140(9): 1955-1967.

395 22. Han S, Cao D, Sha J, Zhu X, Chen D. LncRNA ZFPM2-AS1 promotes lung adenocarcinoma 396 progression by interacting with UPF1 to destabilize ZFPM2. Molecular oncology 2020.

397 23. Dutta P, Sabri N, Li J, Li WX. Role of STAT3 in lung cancer. Jak-stat 2014, 3(4): e999503.

398 24. Mitsudomi T, Yatabe Y. Mutations of the epidermal growth factor receptor gene and related 399 genes as determinants of epidermal growth factor receptor tyrosine kinase inhibitors sensitivity in 400 lung cancer. Cancer science 2007, 98(12): 1817-1824.

401 25. Zhang Y, Zhang J, Xu K, Xiao Z, Sun J, Xu J, Wang J, Tang Q. PTEN/PI3K/mTOR/B7-H1 402 signaling pathway regulates cell progression and immuno-resistance in pancreatic cancer. Hepato403 gastroenterology 2013, 60(127): 1766-1772.

404 26. Pardoll DM. The blockade of immune checkpoints in cancer immunotherapy. Nature reviews 405 Cancer 2012, 12(4): 252-264.

406 27. Rech AJ, Vonderheide RH. Dynamic interplay of oncogenes and T cells induces PD-L1 in the 407 tumor microenvironment. Cancer discovery 2013, 3(12): 1330-1332.

408 28. Taube JM, Anders RA, Young GD, Xu H, Sharma R, McMiller TL, Chen S, Klein AP, Pardoll

409 DM, Topalian SL, Chen L. Colocalization of inflammatory response with B7-h1 expression in 410 human melanocytic lesions supports an adaptive resistance mechanism of immune escape. Science 411 translational medicine 2012, 4(127): 127ra137. 
412 29. Wang QM, Lian GY, Song Y, Huang YF, Gong Y. LncRNA MALAT1 promotes

413 tumorigenesis and immune escape of diffuse large B cell lymphoma by sponging miR-195. Life

414 sciences 2019, 231: 116335.

415 30. Nath AP, Ritchie SC, Grinberg NF, Tang HH, Huang QQ, Teo SM, Ahola-Olli AV, Wurtz P,

416 Havulinna AS, Santalahti K, Pitkanen N, Lehtimaki T, Kahonen M, Lyytikainen LP, Raitoharju

417 E, Seppala I, Sarin AP, Ripatti S, Palotie A, Perola M, Viikari JS, Jalkanen S, Maksimow M, Salmi

418 M, Wallace C, Raitakari OT, Salomaa V, Abraham G, Kettunen J, Inouye M. Multivariate

419 Genome-wide Association Analysis of a Cytokine Network Reveals Variants with Widespread 420 Immune, Haematological, and Cardiometabolic Pleiotropy. American journal of human genetics 421 2019, 105(6): 1076-1090.

422

423

424

425

426

427

428

430

431

432

433

434

435

436

437

\section{Figure legends}

Figure 1. Correlation between higher ZFPM2-AS1 expression and poor prognosis. (A) Scatter plot of ZFPM2-AS1 expression values in NSCLC and normal tissue samples from the RNA-Seq dataset of TCGA (y-axis represents the FPKM value, **** means $\mathrm{p}<0.0001)$. (B) AUC value of the ROC curve based on ZFPM2-AS1 expression values. (C) The Kaplan-Meier survival curve with its corresponding log-rank test of ZFPM2-AS1.

Figure 2. ZFPM2-AS1 upregulation in NSCLC samples and cell lines. The expression levels of samples and cell lines were determined using quantitative RT-PCR. All data analyses were performed using the mean values of individual tissues or the mean value \pm SD of each cell line from three independent experiments. (A) Differential ZFPM2-AS1 expression across 50 NSCLC pairs and adjacent normal tissues. An unpaired Student's t-test was used to find this statistical result. (B) Differential ZFPM2-AS1 expression in individual cell lines. The statistical result was found using ANOVA (parametric) test. * $\mathrm{P}<0.05, * * \mathrm{P}<0.01, * * * \mathrm{P}<0.001, * * * * \mathrm{P}<0.0001$. 
439 Figure 3. The positive correlation between ZFPM2-AS1 and NSCLC cell proliferation. (A) 440 qRT-PCR was used to evaluate the knockdown efficiency (24h) of ZFPM2-AS1 with the specific 441 siRNAs and scrambled target sequences. The y-axis value represented the ratio of ZFPM2-AS1 442 expression in siRNA groups to that of the control group. GAPDH was used as the reference gene.

443 (B) The MTT assay was performed so that the ZFPM2-AS1 knockdown inhibited H460 cell 444 proliferation. The statistical data were analyzed using an unpaired Student's t-test. (C) ZFPM2445 AS1 knockdown inhibited A549 cell proliferation with and without IFN- $\gamma$. (D-G) Colony 446 formation assays were performed to illuminate A549 and H460 cell proliferation upon ZFPM2447 AS1 knockdown. (H) The effect of ZFPM2-AS1 overexpression on A549 cell proliferation with 448 and without IFN- $\gamma . * \mathrm{P}<0.05 . * * \mathrm{P}<0.01 . * * * \mathrm{P}<0.001 . * * * * \mathrm{P}<0.0001$.

Figure 4. The positive correlation between ZFPM2-AS1 and the migration and invasion of NSCLC cells. (A-D) ZFPM2-AS1 knockdown inhibited the motility of A549 and H460 cells by the wound healing assay. (E) (F) The transwell assay was performed to determine whether ZFPM2AS1 knockdown inhibited A549 and H460 cell invasion. (G-J) In A549 cells, ZFPM2-AS1 silencing decreased the invasion and migration potential with and without IFN- $\gamma(48 \mathrm{~h})$. (K-N) The impact of ZFPM2-AS1 overexpression on the invasion and migration ability of A549 cells with and without IFN- $\gamma(48 \mathrm{~h}) .{ }^{*} \mathrm{P}<0.05 . * * \mathrm{P}<0.01 .{ }^{*} * * \mathrm{P}<0.001$.

Figure 5. The location and effect on the ZFPM2-AS1 cell cycle. (A) The ZFPM2-AS1 expression levels in the A549 cell nucleus and cytoplasm fractions were found using qRT-PCR. The statistical Chi-square test was performed with three independent experiments. (B-I) We analyzed the flow cytometric of the cell cycle $24 \mathrm{hrs}$ after ZFPM2-AS1 knockdown or overexpression in the presence or absence of IFN- $\gamma$. 
464 Figure 6. Correlations between ZFPM2 expression and immune infiltration level. (A) ZFPM2

465 expression levels of various human tumor types were validated using TIMER $(* \mathrm{p}<0.05, * * \mathrm{p}<0.01$, $466 * * * \mathrm{p}<0.001)$. The color red signified tumor tissues, blue signified normal tissues, and purple 467 signified metastasis tissue. (B) The correlations between ZFPM2 expression and tumor purity, B 468 cells, CD8+ T cells, CD4+ T cells, macrophages, neutrophils, or dendritic cells were analyzed.

469

470

Figure 7. ZFPM2-AS1 positively regulated PD-L1 expression via the JAK-STAT and AKT pathways. (A, B) ZFPM2-AS1 was downregulated by three individual siRNAs. The relevant proteins were identified by Western blot during ZFPM2-AS1 knockdown (C, D) or overexpression, (E, F) with or without IFN- $\gamma$ treatment.

Table 1. The association between ZFPM2-AS1 expression and clinical measures in LUAD 477 patients.

Additional files

Table S1: Specific primers for qRT-PCR and siRNA Smart Silencer sequences. 


\section{Table $\mathbf{1}$ (on next page)}

Table1

The association between ZFPM2-AS1 expression and clinical measures in LUAD patients 
1

2 Table 1. The association between ZFPM2-AS1 expression and clinical measures in LUAD

3 patients.

\begin{tabular}{|c|c|c|c|c|}
\hline \multirow{2}{*}{ Characteristics } & \multirow{2}{*}{$\mathrm{N}$} & \multicolumn{3}{|c|}{ Relative ZFPM2-AS1 expression } \\
\hline & & Low & High & $P$ value \\
\hline \multicolumn{5}{|l|}{ Age(years) } \\
\hline$>65$ & 11 & 5 & 6 & \multirow{2}{*}{0.733} \\
\hline$\leq 65$ & 39 & 20 & 19 & \\
\hline \multicolumn{5}{|l|}{ Gender } \\
\hline Male & 24 & 14 & 10 & \multirow{2}{*}{0.258} \\
\hline Female & 26 & 11 & 15 & \\
\hline
\end{tabular}

Differentiation

$\begin{array}{cccc}\text { Well,moderate } & 38 & 19 & 19 \\ \text { Poor } & 12 & 6 & 6\end{array}$

19

6

0.999

Tumor size(maximum diameter)

$\begin{array}{lcccc}>3 \mathrm{~cm} & 33 & 12 & 21 & 0.007^{* *} \\ \leq 3 \mathrm{~cm} & 17 & 13 & 4 & \end{array}$

Histological tumor type

Squamous cell carcinoma

Adenocarcinoma

30

17

0.248

Smoking history

\section{Smokers}

Never smokers

Lymph node metastasis

Positive
20

30

23
11

14

16

9

16

0.564

$12 \quad 0.777$ 
$\begin{array}{llll}\text { Negative } & 27 & 14 & 13\end{array}$

TNM stage

$\begin{array}{ccccc}1 \text { stage } & 23 & 15 & 8 & 0.047^{*} \\ 2.3 .4 \text { stage } & 27 & 10 & 17 & \end{array}$

4 Data were analyzed by Chi-square. Median value: 5.04 .

$5 * \mathrm{P}<0.05, * * \mathrm{P}<0.01$. Data were analyzed by Chi-square test. The high and low groups were

6 divided by the median expression value of ZFPM2-AS1 (5.04).

7 


\section{Figure 1}

Figure1

Correlation between higher ZFPM2-AS1 expression and poor prognosis.

A

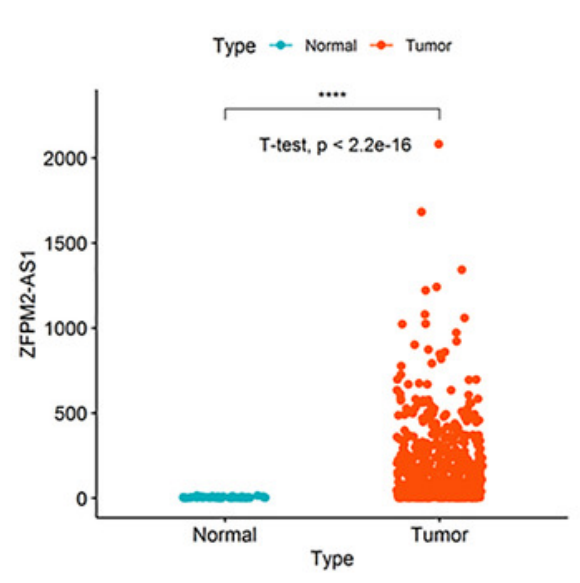

B

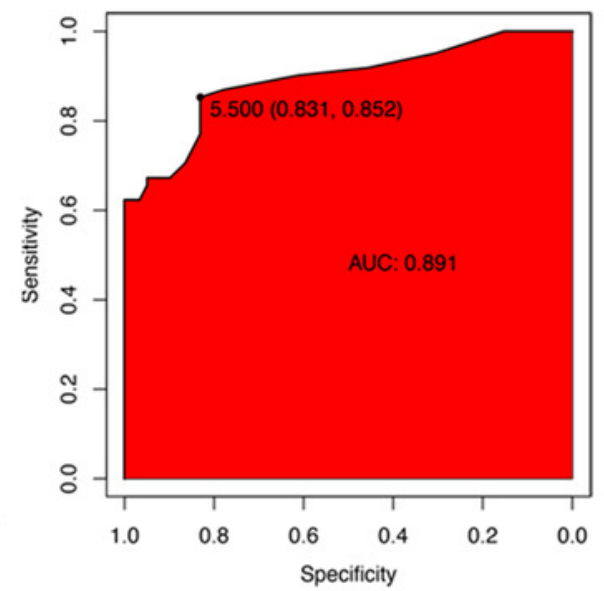

C

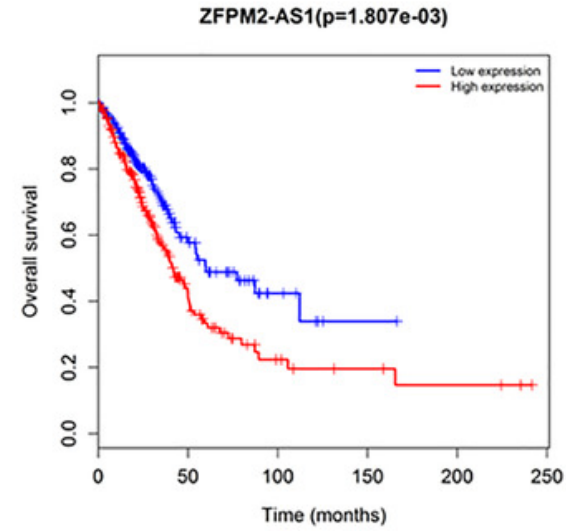


Figure 2

Figure2

ZFPM2-AS1 upregulation in NSCLC samples and cell lines.

A

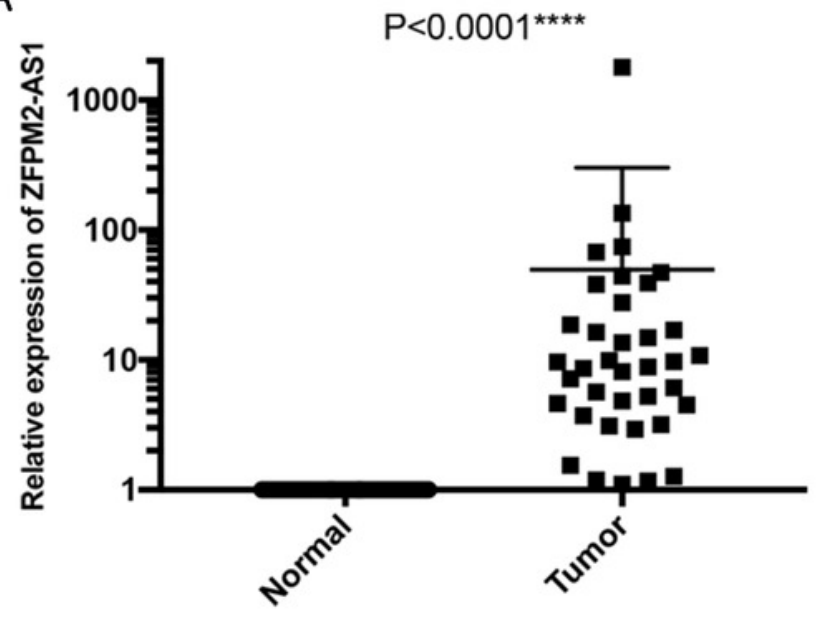

B

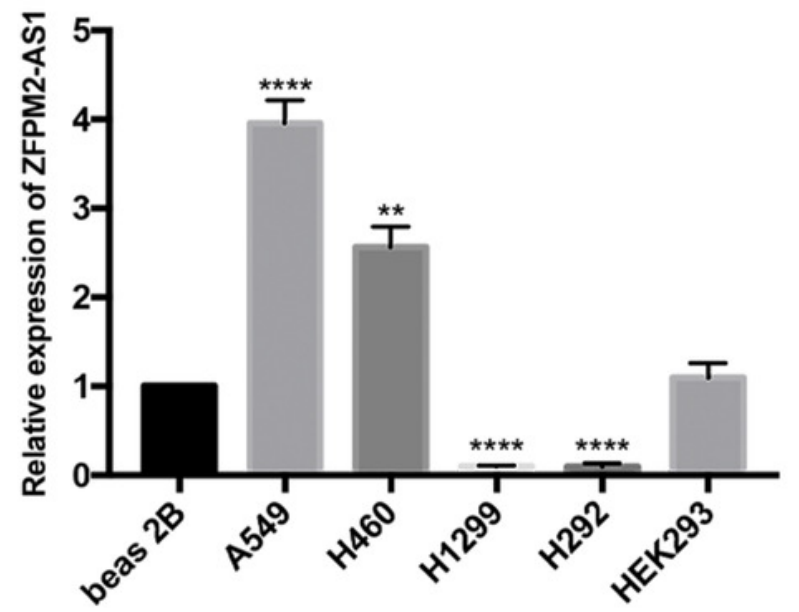


Figure 3

Figure3

The positive correlation between ZFPM2-AS1 and NSCLC cell proliferation.

A

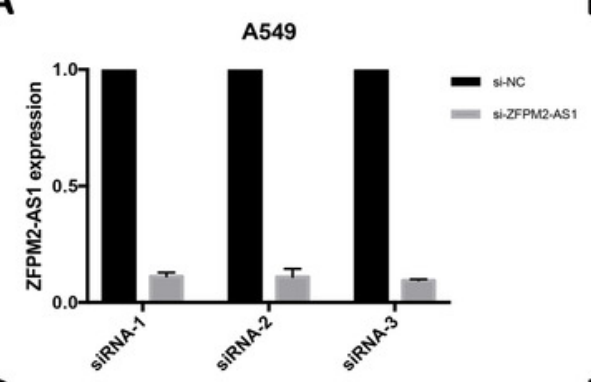

D

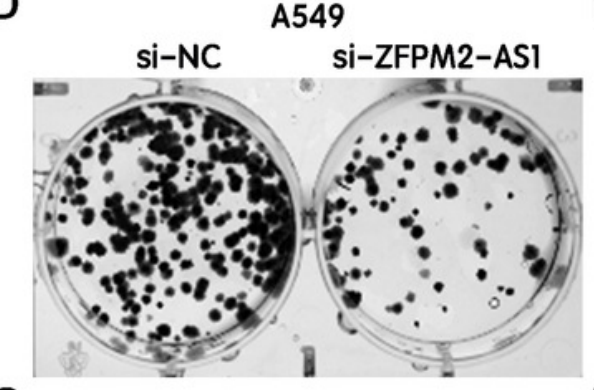

G

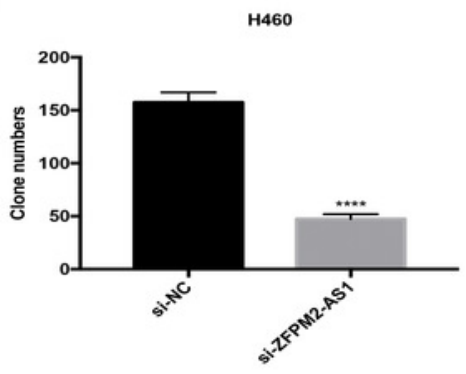

B

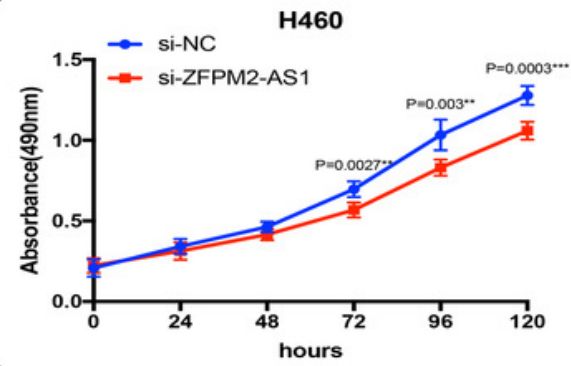

E

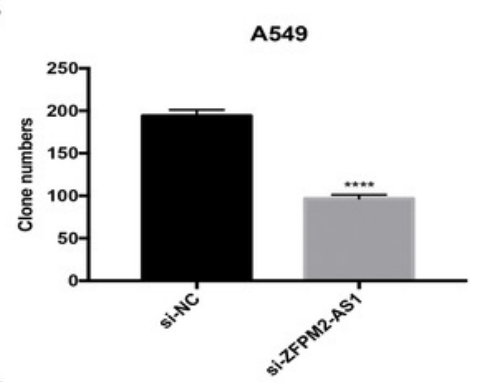

$\mathrm{H}$

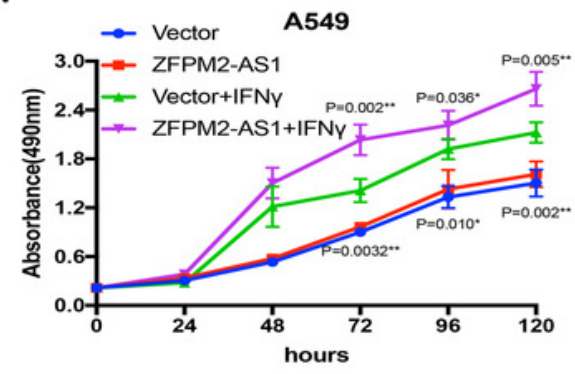

C

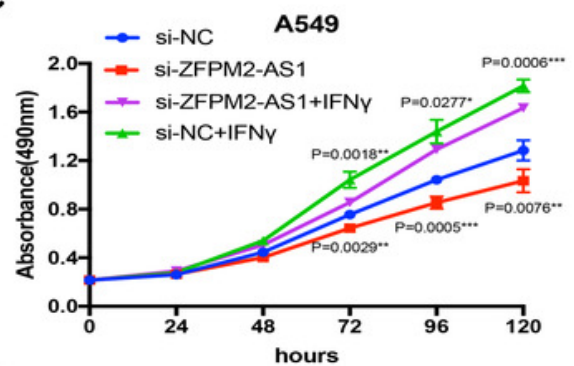

$\mathbf{F}$

$\mathrm{H} 460$

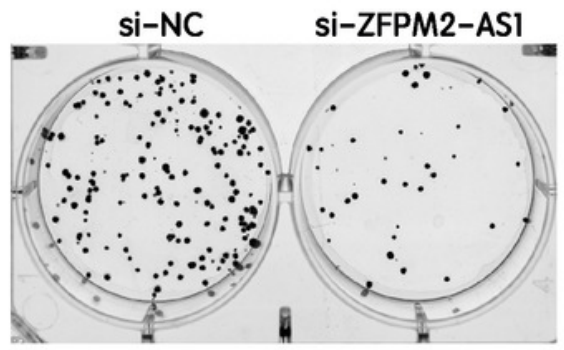


Figure 4

Figure4

The positive correlation between ZFPM2-AS1 and the migration and invasion of NSCLC cells.

A

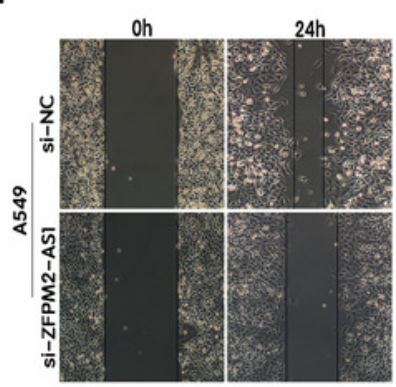

E

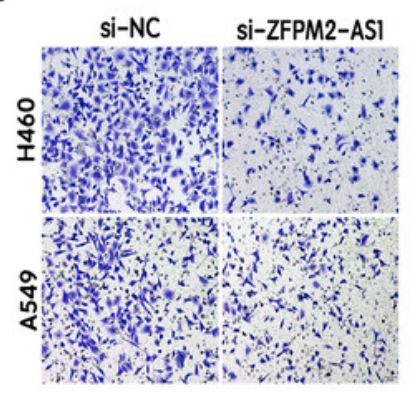

I

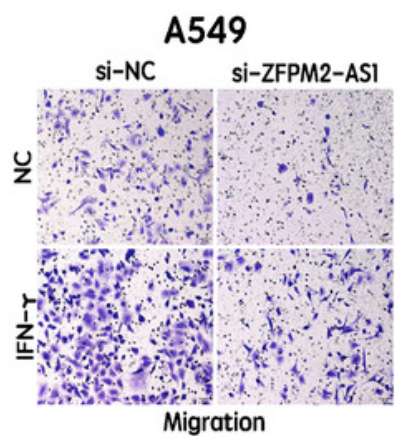

M

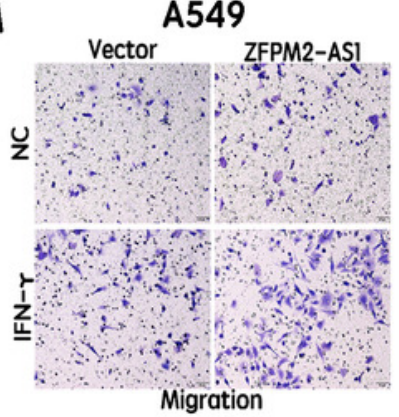

B

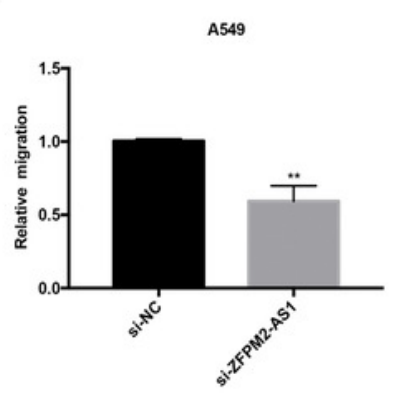

$\mathbf{F}$

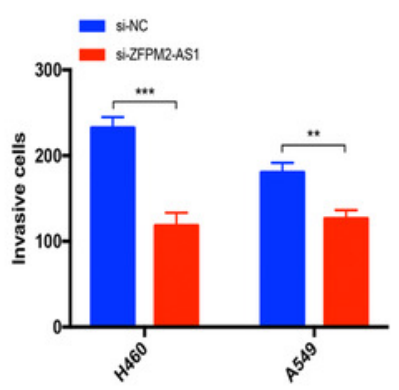

J

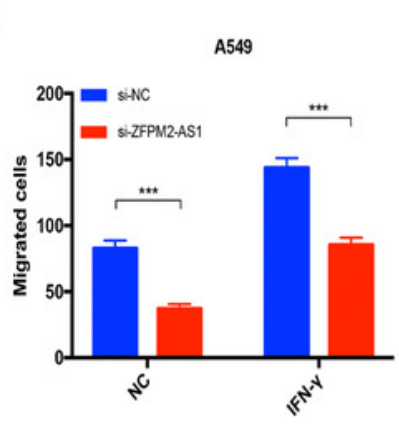

$\mathbf{N}$

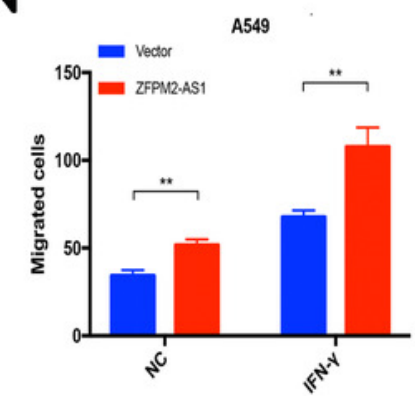

C

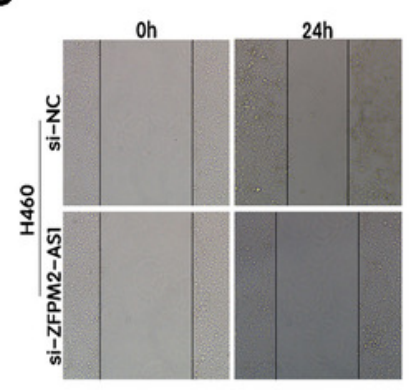

G

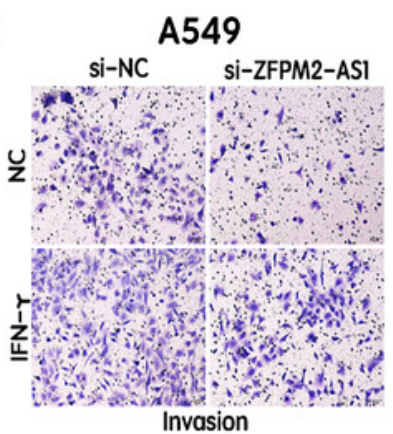

K

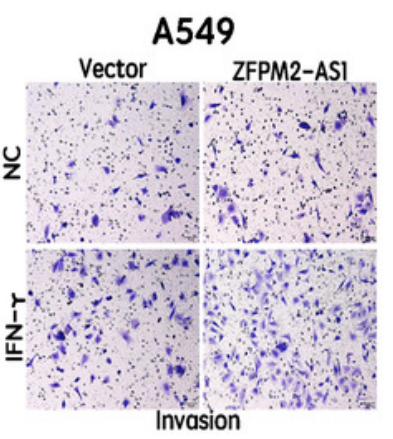

D

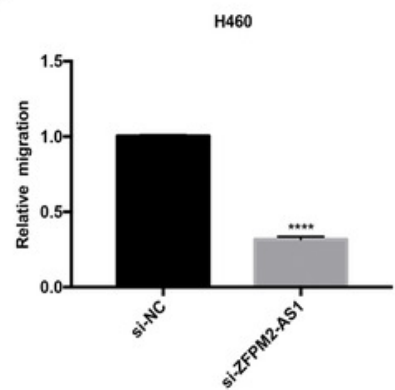

H

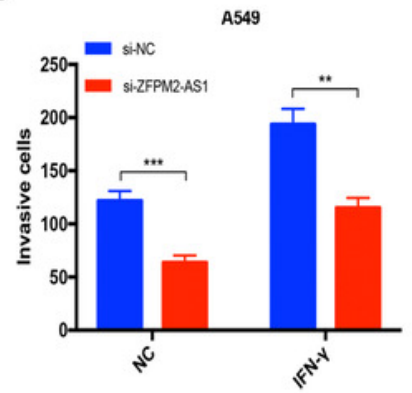

L

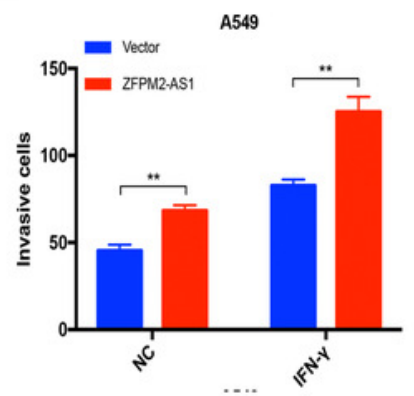


Figure 5

Figure5

The location and effect on the ZFPM2-AS1 cell cycle.

A

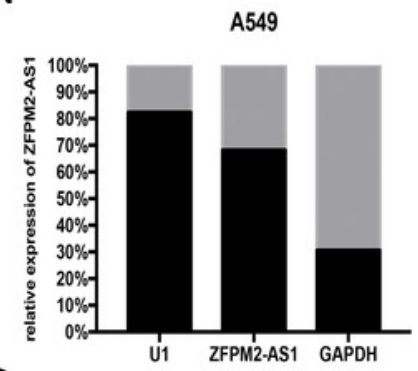

D

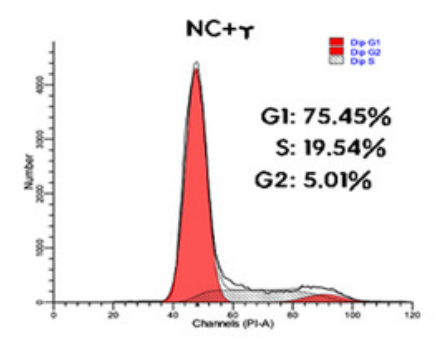

G

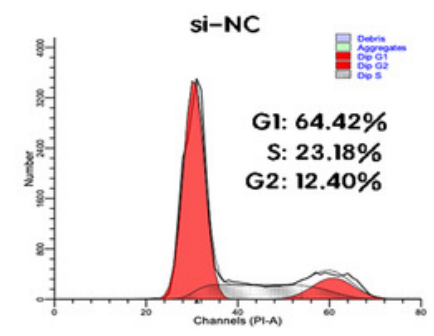

B

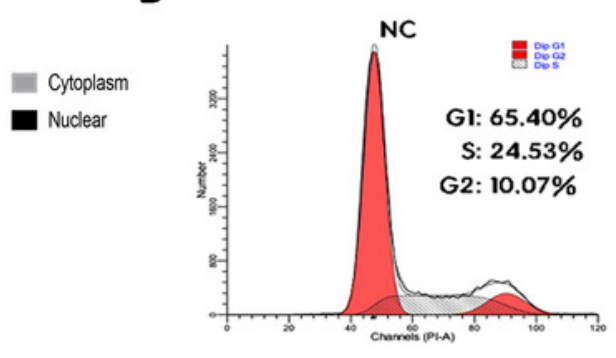

E

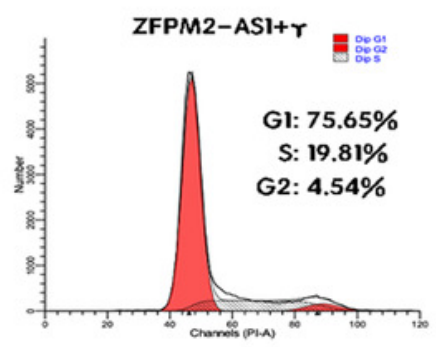

$\mathrm{H}$

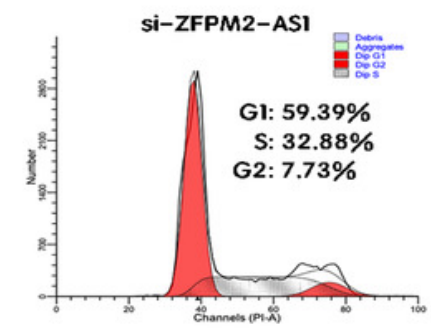

C

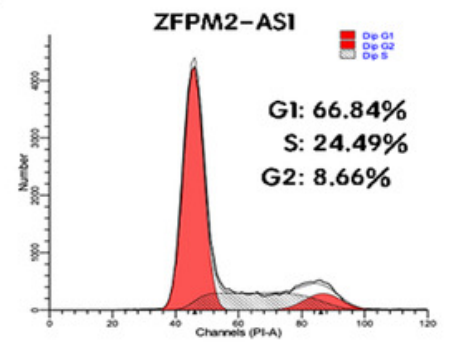

$\mathrm{F}$

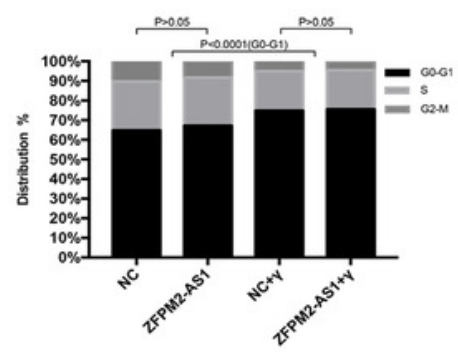

I

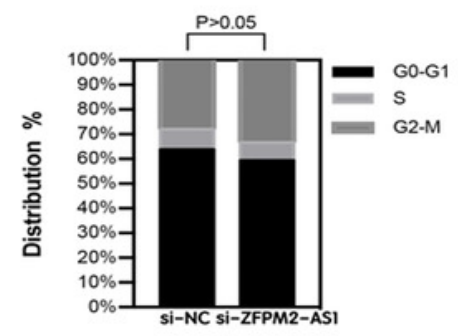


Figure 6

Figure6

Correlations between ZFPM2 expression and immune infiltration level.
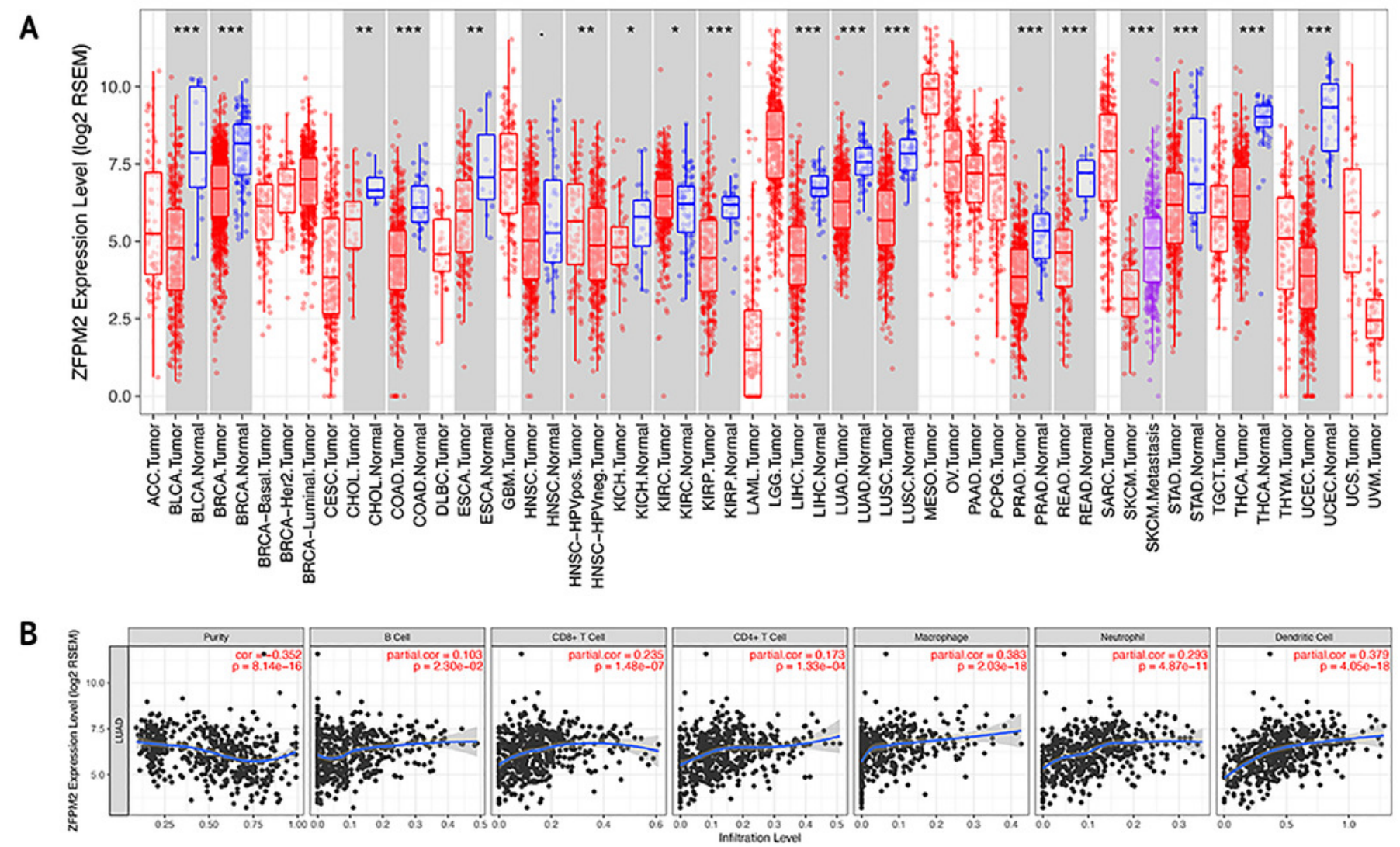
Figure 7

Figure7

ZFPM2-AS1 positively regulated PD-L1 expression via the JAK-STAT and AKT pathways. 
A

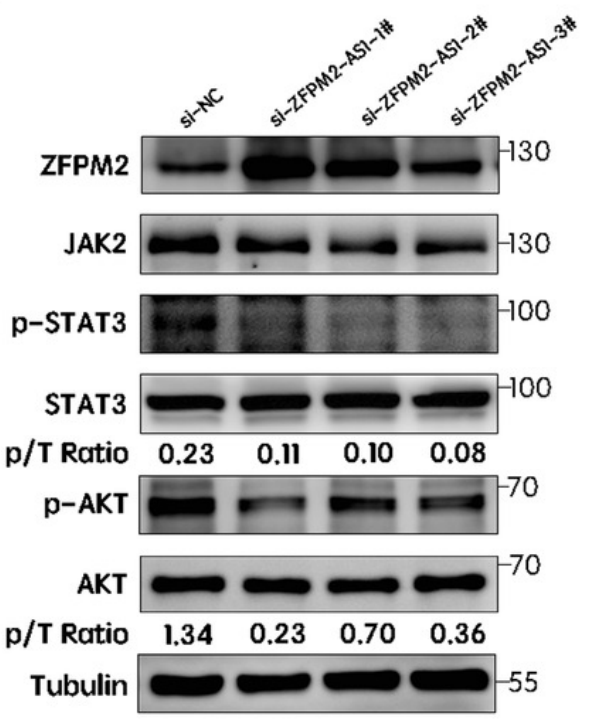

C

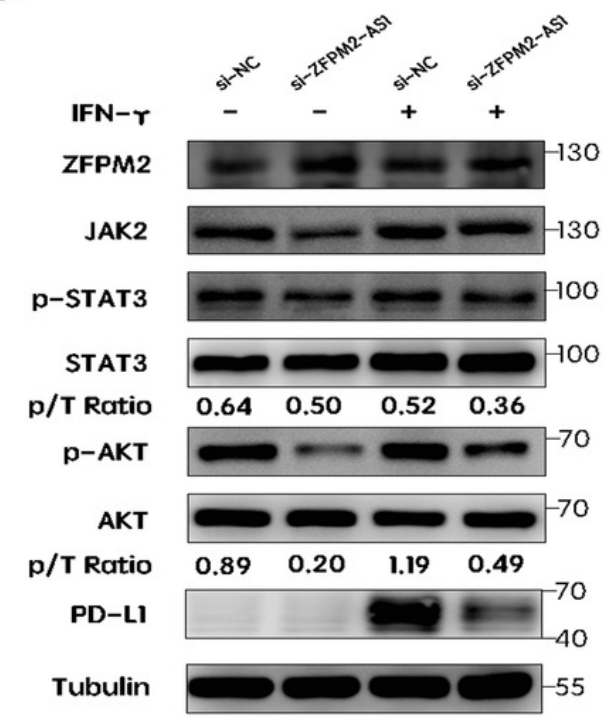

E

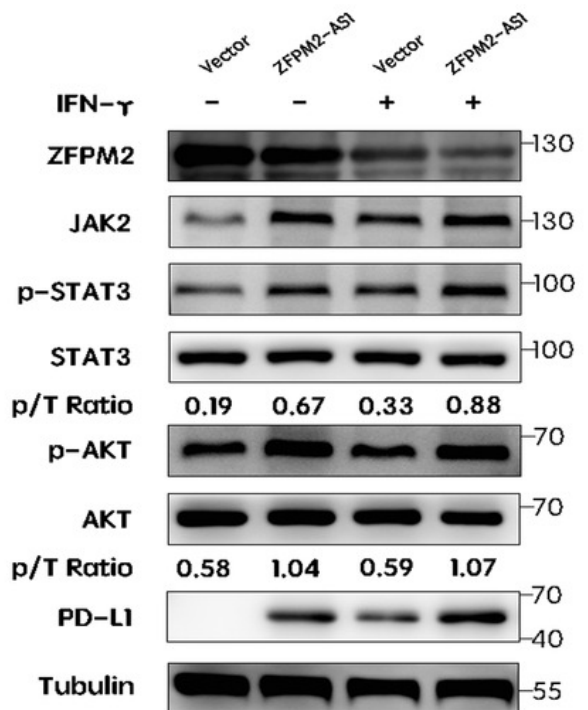

B

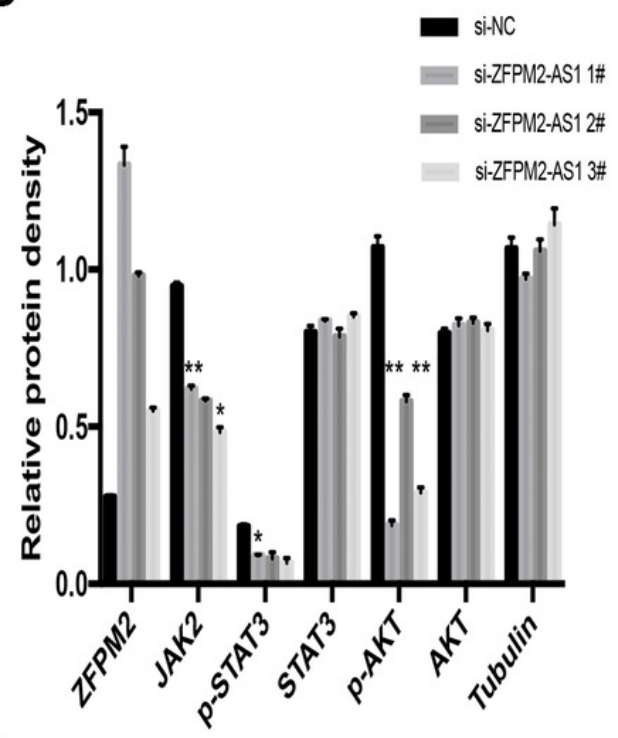

D

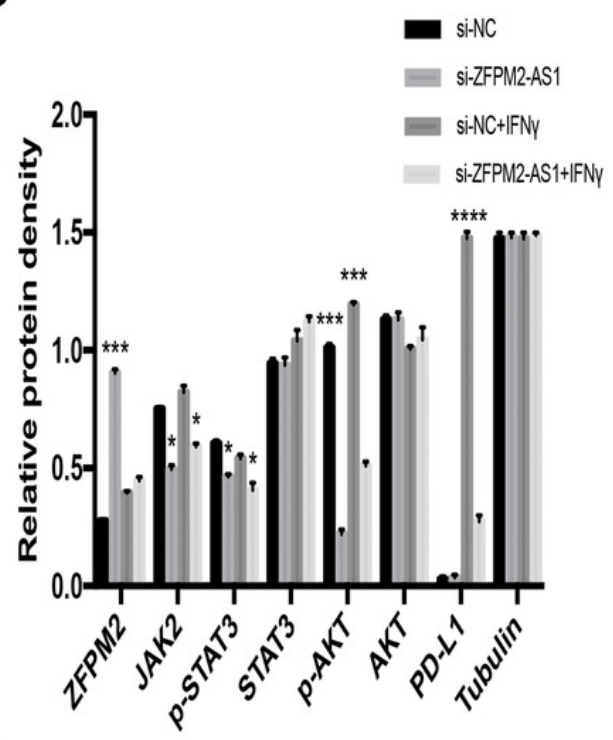

$F$

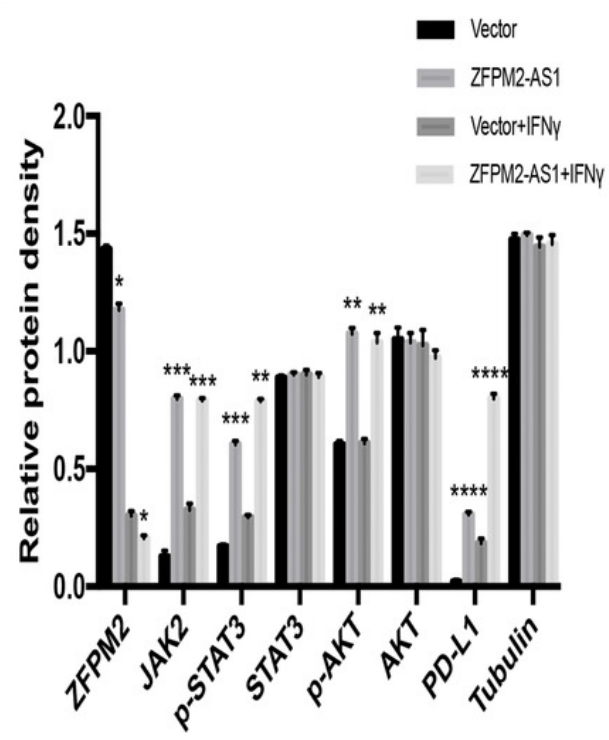

PeerJ reviewing PDF | (2020:04:48469:2:0:NEW 12 Sep 2020) 